\title{
Pump-Induced Dynamical Tunneling in a Deformed Microcavity Laser
}

\author{
Juhee Yang, ${ }^{1}$ Sang-Bum Lee, ${ }^{2}$ Songky Moon, ${ }^{1}$ Soo-Young Lee, ${ }^{1}$ Sang Wook Kim, ${ }^{3}$ Truong Thi Anh Dao, ${ }^{1}$ \\ Jai-Hyung Lee, ${ }^{1}$ and Kyungwon $\mathrm{An}^{1, *}$ \\ ${ }^{1}$ Department of Physics and Astronomy, Seoul National University, Seoul 151-742, Korea \\ ${ }^{2}$ Korea Research Institute of Standards and Science, Daejeon 305-600, Korea \\ ${ }^{3}$ Department of Physics Education, Pusan National University, Busan 609-735, Korea
}

(Received 17 December 2009; published 14 June 2010)

\begin{abstract}
Pump-induced dynamical tunneling has been observed in free-space resonant optical pumping of a deformed microcavity by employing excitation spectroscopy. A focused-pump beam was injected into the cavity by refraction and then coupled to a high- $Q$ cavity mode via dynamical tunneling. Pump-coupling efficiency as high as $50 \%$ and an effective coupling constant responsible for the tunneling were obtained from the observed pumping efficiency with a mode-mode coupling model.
\end{abstract}

DOI: 10.1103/PhysRevLett.104.243601

Efficient optical pumping of high- $Q$ cavity modes is of primary concern in microcavity applications such as microlasers and optoelectronic components and devices [1]. Prism and tapered-fiber couplings are commonly used for ultrahigh- $Q$ whispering gallery modes [2,3]. However, simple free-space coupling without near-field couplers is often needed for practical reasons such as experimental limitations or implementation cost. Microcavities deformed from rotational symmetry can provide directional emission of high- $Q$ modes, and, moreover, allow one to excite them by a free-space pump beam. Recently, cryogenic cooling of an optomechanical resonator assisted by free-space evanescent coupling has been reported in a slightly deformed microsphere [4]. Efficient nonresonant optical pumping based on ray chaos has also been demonstrated in deformed microcavities [5,6], suggesting further enhancements by resonant optical pumping.

In a significantly deformed microcavity, the ray dynamics typically exhibits both regular and chaotic trajectories [7], resulting in a mixed phase space: high- $Q$ modes are usually localized on regular regions such as invariant tori and regular islands surrounded by a chaotic sea. It is known that light in high- $Q$ modes localized on those regular regions can be transferred to the nearest chaotic sea by a dynamical process and then transported by ray chaos resulting in a directional refractive output. The dynamical process is called dynamical tunneling [8] since the transport from a regular region to the chaotic sea is strictly forbidden classically.

The dynamical tunneling is thus an essential link between high- $Q$ modes and directional output, important assets for the aforementioned photonic applications. In fact, dynamical tunneling is of great current interest and has been studied theoretically in various physical systems [9-14]. Experimental signatures of dynamical tunneling were reported in a microwave billiard in energy splitting $[15,16]$, in an acoustical resonator through spectral statistics [17], and in a cold atomic sample in atomic momentum distribution $[18,19]$. In microcavities, although it has been
PACS numbers: 42.55.Sa, 03.65.Xp, 05.45.Mt, 42.50.-p

studied theoretically [20-23], there is no direct experimental evidence for dynamical tunneling.

How can then the dynamical tunneling be used to improve the optical pumping? The answer lies in the reverse of the aforementioned output process. If we inject a pump beam in a time reversed way, the pump light might be transferred to high- $Q$ modes via dynamical tunneling. Since the tunneling is a wave-mechanical process, it would occur when the pump is resonant with the target mode. However, it is not clear how efficient the process would be. There is neither measurement nor calculation on such pump-induced dynamical tunneling processes.

In this Letter, we report the first experimental observation of pump-induced dynamical tunneling phenomena in a microcavity laser by using excitation spectroscopy. High- $Q$ cavity modes in a significantly deformed microcavity were resonantly pumped by the pump-induced dynamical tunneling process. The pumping efficiency (lasing threshold) was enhanced (lowered) on resonance by 2 orders of magnitude from that of the nonresonant pumping based on chaotic ray dynamics. Using a mode-mode coupling model on chaotic modes and a high- $Q$ mode, we obtained the resonant pump-coupling efficiency as well as an effective coupling constant responsible for the tunneling from the observed pumping efficiency. The resulting coupling efficiencies are surprisingly high, about $50 \%$ for the observed high- $Q$ modes.

Our experiment was done on a two-dimensional high- $Q$ microcavity made of a liquid jet of ethanol doped with Rhodamine- 640 dye at a concentration of $0.02 \mathrm{mM} / 1$ as gain molecules. The technical details of our liquid-jet microcavity are described elsewhere [24]. The cavity shape was measured [25] to be a quadru-octupole approximated by the following equation in the polar coordinates: $r(\phi) \simeq$ $a_{0}\left(1+\eta \cos 2 \phi+\epsilon \eta^{2} \cos 4 \phi\right)$, where $a_{0} \simeq 15 \pm 0.1 \mu \mathrm{m}$, the mean radius of the cavity, and $\eta=0.190 \pm 0.005$ the deformation parameter with $\epsilon=0.42 \pm 0.05$.

Figure 1(a) shows the mode spectrum of our deformed microcavity. It was obtained by measuring the fluorescence 

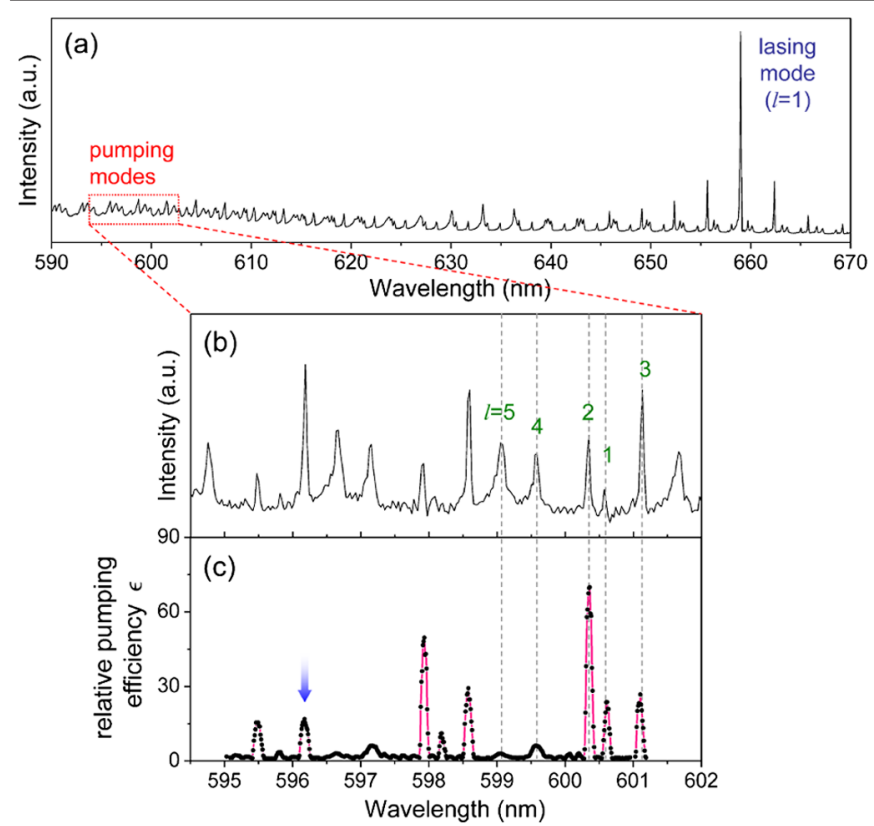

FIG. 1 (color online). (a) Mode spectrum of our microcavity. (b) Magnified view around $600 \mathrm{~nm}$. (c) Pumping-efficiency spectrum obtained by the excitation spectroscopy explained in the text. Each data point represents an averaged value over several measurements with about $15 \%$ error.

or lasing emission of the dye-doped microcavity with a spectrometer when the cavity was flood pumped from the side with a wide beam of a $532 \mathrm{~nm} \mathrm{Nd:Vanadate} \mathrm{laser.} \mathrm{We}$ can identify five distinct mode groups with mode orders $l=1,2,3,4,5$ as shown in Fig. 1(b), a magnified view of the spectrum in the region of $600 \mathrm{~nm}$ [26]. Each of the mode groups has a regular mode spacing, supporting that the observed modes are localized on periodic orbits. Furthermore, the observed high- $Q$ factors of modes $1,2,3$ (order of $10^{5}-10^{6}$ ) indicate that they are regular modes localized on stable periodic orbit. The modes 4 and 5 with relatively low $Q$ factors (order of $10^{4}-10^{3}$ ) are scarred modes localized on unstable periodic orbit [27].

The basic idea of our experiment is as follows. We first inject a (free-space) collimated pump beam into the deformed microcavity by refraction and adjust its incident angle $\theta$ and displacement $X$ defined in Fig. 2 until the nonresonant pumping efficiency for a particular lasing mode (let us call it $L$ mode) is maximized [6]. Then, we scan the pump wavelength and look for any resonance effect, e.g., a sudden enhancement in the lasing power, in a manner of excitation spectroscopy.

For this experiment, a frequency-tunable dye laser (Coherent CR-590) with a full linewidth $2 \gamma_{L}$ of $0.025 \mathrm{~nm}$ near wavelength $600 \mathrm{~nm}$ provided a pump beam, which was focused down to a beam waist of $0.8 \mu \mathrm{m}$ on the cavity column from the side and could be translated by an arbitrary displacement $X$. Pumping efficiency was measured from the threshold pump power for the $L$ mode denoted in Fig. 1(a) by using the fact that the
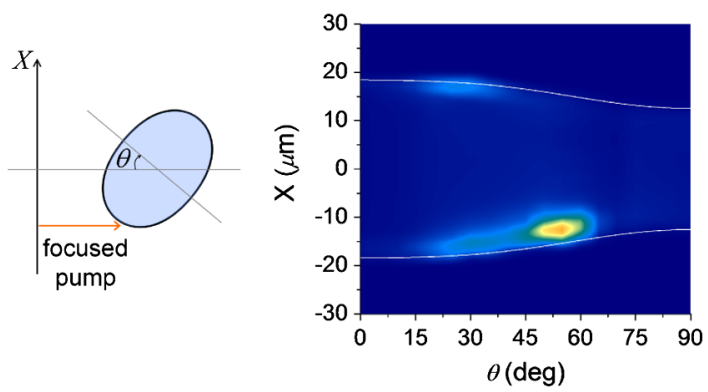

FIG. 2 (color online). Pumping-efficiency spatial distribution in $\theta$-X space with the pump wavelength fixed at $l=3 P$-mode resonance indicated by a blue arrow in Fig. 1(c). The left-hand figure shows the cross section of the liquid jet.

pumping efficiency is inversely proportional to the threshold pump power.

When we scanned the pump wavelength, we observed the output power of the $L$ mode going up whenever the pump wavelength became resonant with a high- $Q$ cavity mode (let us call it $P$ mode) in the mode spectrum of Fig. 1(b). What happened is that the pump laser must have been resonantly coupled to the $P$ mode and the resulting high intensity in that mode effectively excited gain molecules there. These molecules would in turn radiatively coupled to the $L$ mode at the longer wavelength which has a substantial spatial overlap with the $P$ mode. Relative pumping efficiency $\epsilon$, obtained from the threshold pump power, with respect to that of the off-resonance pumping as a function of the pump wavelength is plotted in Fig. 1(c). Remarkably, the pumping efficiency on resonance is enhanced by up to 70 times from that of the nonresonant pumping case (base line) [6]. This large enhancement in free-space pumping has an important implication related to photonics applications.

The resonance-enhanced pumping efficiency of Fig. 1(c) exhibits a $\theta-X$ dependence as shown in Fig. 2, where solid white lines indicate the projected edges of the cavity as $\theta$ is varied. For this measurement the pump wavelength was fixed on resonance with the $l=3 P$ mode marked by a blue arrow in Fig. 1(c). In fact, the same $\theta-X$ dependence was observed for the other $P$ modes and even at off resonance as well, which is consistent with the mode-independent farfield patterns shown in Ref. [26]. A maximum pumping efficiency occurs at $(\theta, X)=\left(55^{\circ},-13 \mu \mathrm{m}\right)$, located well inside the cavity boundary, indicating that the pump beam must have been injected into the cavity by refraction, not by evanescent tunneling. The pump beam would then undergo chaotic ray dynamics inside the cavity. This reasoning suggests that the observed resonant pumping must have been mediated by a classically forbidden process transporting the pump light from the chaotic sea to the regular region, which is just the dynamical tunneling.

For close examination of this process, it is convenient to use a phase-space diagram as shown in Fig. 3, where high- $Q$ modes with $l=1,2,3$ are localized on stable 


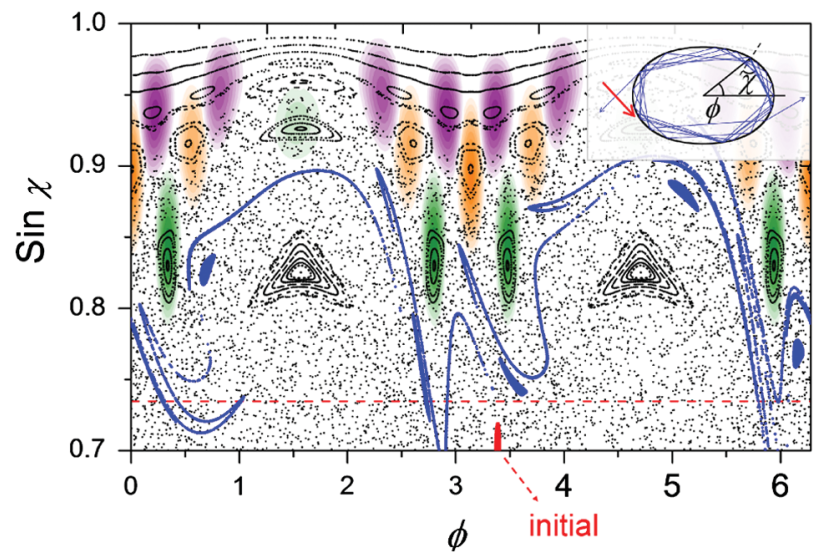

FIG. 3 (color). Phase space (Poincaré surface of section) for our deformed microcavity is presented in the Birkhoff's coordinates, in which a ray is reflected off the cavity boundary at polar angle $\phi$ with an incidence angle $\chi$. Husimi distributions of $l=1,2,3$ modes are shown in purple, orange, and green, respectively. The red-filled circle indicates the initial bundle of rays and blue lines represent the subsequent pump-beam trajectories. Inset: real-space pump-beam trajectories.

periodic orbits (island structures). These regular structures are well overlapped with their wave-function phase-space distributions or the Husimi distributions plotted in purple, orange, and green, respectively. A bundle of rays initially prepared in a region (colored red) around $(\phi, \sin \chi) \simeq$ $(\pi, 0.71)$, corresponding to the maximum pumpingefficiency position in Fig. 2, will follow the chaotic trajectories shown as blue curves in Fig. 3 (phase space) and in the inset (real space). These pump trajectories are located in the chaotic sea, classically disconnected from the regular regions. Dynamical tunneling must then occur from the chaotic sea to the stable islands in order to fulfill resonant excitation of the high- $Q$ modes.

We can model the dynamical tunneling as a mode-mode coupling process as depicted in Fig. 4(a). The chaotic sea is modeled as a collection of a large number of very-low- $Q$ uncoupled chaotic modes. These chaotic modes are driven by an external pump-laser with an electric-field amplitude $E_{0}$ and then by dynamical tunneling processes these modes are coupled to an uncoupled high- $Q$ regular mode of decay rate $\gamma_{r}$. The decay rate of $n$th chaotic mode is $\gamma_{n}$ and its coupling constant to the high- $Q$ mode is $g_{n}$ (assumed real, $n=1,2,3, \ldots, N)$, satisfying $\left|g_{n}\right|, \gamma_{r} \ll \gamma_{n}$. Effects of partial barriers are included in $g_{n}$ and $\gamma_{n}$. Here, the uncoupled modes are not true eigenmodes of the system since the coupling between them are treated separately in our model-the term "mode" in the following theoretical description actually means an uncoupled mode if not noted otherwise.

With $\mathcal{E}_{n}$ and $\mathcal{E}_{r}$ denoting the slowly varying electric-field envelopes of the $n$th chaotic mode and the regular mode, respectively, the equations of motion describing the modemode coupling processes are then
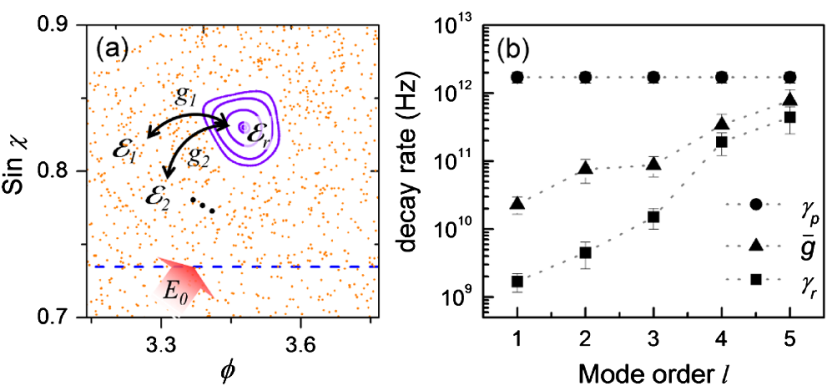

FIG. 4 (color online). (a) Mode-mode coupling model. Uncoupled chaotic mode $\mathcal{E}_{n}$, excited by refractively injecting a pump laser $E_{0}$, can couple to an uncoupled regular mode $\mathcal{E}_{r}$ with a coupling constant $g_{n}$ by dynamical tunneling. (b) Decay rate $\gamma_{r}$ and the effective coupling constant $\bar{g}$ obtained from the pumping-efficiency data for each high- $Q$ cavity mode.

$$
\begin{gathered}
\dot{\mathcal{E}}_{n}+\gamma_{n} \mathcal{E}_{n}=a_{n} E_{0}-g_{n} \mathcal{E}_{r}, \\
\dot{\mathcal{E}}_{r}+\gamma_{r} \mathcal{E}_{r}=\sum_{n}^{N} g_{n} \mathcal{E}_{n},
\end{gathered}
$$

where $a_{n}$ is the coupling coefficient of the external pumplaser field into the $n$th chaotic mode and we assume that the pump laser is resonant with the regular mode for simplicity. More general equations including pump detuning are considered and solved in Ref. [28]. We quote only the main results below.

The steady-state on-resonance pumping efficiency $\epsilon$ relative to that of off-resonance pumping is given by

$$
\epsilon= \begin{cases}(1-\alpha)+\frac{\gamma_{p} \beta_{r}}{\gamma_{r}^{\prime} \beta_{p}} \alpha^{\prime}, & \text { for } \gamma_{L} \ll \gamma_{r}^{\prime}, \\ \left(1-\frac{\gamma_{r}^{\prime}}{\gamma_{L}} \alpha\right)+\frac{\gamma_{p} \beta_{r}}{\gamma_{L} \beta_{p}} \alpha^{\prime}, & \text { for } \gamma_{L} \gg \gamma_{r}^{\prime},\end{cases}
$$

where $\alpha=\frac{G(2+G)}{(1+G)^{2}}, \alpha^{\prime}=\frac{G}{1+G}, G=\sum_{n} \frac{g_{n}^{2}}{\gamma_{r} \gamma_{n}}, \gamma_{r}^{\prime}=\gamma_{r}(1+$ $G)$, and $\gamma_{L}$ is the pump-laser half linewidth. The decay rate $\gamma_{p}$ is defined as

$$
\frac{\bar{g}^{2}}{\gamma_{p}} \equiv \sum_{n} \frac{g_{n}^{2}}{\gamma_{n}}
$$

where $\bar{g} \equiv \sum_{n} g_{n} \mathcal{E}_{n}^{0} / \sqrt{\sum_{n}\left|\mathcal{E}_{n}^{0}\right|^{2}}$ and $\mathcal{E}_{n}^{0}\left(\equiv \frac{a_{n}}{\gamma_{n}} E_{0}\right)$ is the offresonance steady-state amplitude of the $n$th chaotic mode excited by the pump laser. Analogous to the enhanced spontaneous emission in the bad cavity limit of the cavity quantum electrodynamics [29], the right-hand side of Eq. (4), equal to $\gamma_{r} G$, is interpreted as the total tunneling rate from the regular mode to all chaotic modes. We can then treat the collection of all chaotic modes as a single pump mode with $\bar{g}$ acting as an effective coupling constant between the pump mode and the regular mode and the rate $\gamma_{p}$ as an effective decay rate of the pump mode when seen by the regular mode [28]. Parameters $\beta_{p}$ and $\beta_{r}$ are the spatial overlap factors of the pump and the regular modes with the $L$ mode of interest, respectively,

By solving the eigenvalue problem associated with Eqs. (1) and (2) with $\dot{\mathcal{E}}_{r}=\dot{\mathcal{E}}_{n}=0$, one can show that the decay rate of the uncoupled regular mode $\gamma_{r}$ is changed to 
$\gamma_{r}^{\prime}=\gamma_{r}+\sum_{n} g_{n}^{2} / \gamma_{n}$ due to its coupling to all uncoupled chaotic modes [28]. In experiment, the quantity measured is not $\gamma_{r}$, but $\gamma_{r}^{\prime}$, inversely proportional to the observed quality factor of the regular eigenmode [26].

The parameter $\alpha$ in Eq. (3) can be interpreted as the fraction of the intracavity pump power of nonresonant pumping that is reduced in the case of the resonant pumping. Therefore, we can identify $\alpha$ as the resonant pumpcoupling efficiency, similar to the pump-coupling efficiency in the usual tapered-fiber evanescent coupling.

Among many variables and parameters in Eq. (3) the only unknowns are $\gamma_{r}$ and $G$. All the others are either measurable or calculable. First, the decay rate $\gamma_{r}^{\prime}$ of each eigenmode $(l=1,2,3,4,5)$ was obtained from the observed $Q$ values, as summarized in Table I. The effective decay rate $\gamma_{p}=1.7 \times 10^{12} \mathrm{~s}^{-1}$ of the pump mode was calculated by ray simulations, considering the average survival path length of a bundle of pump rays injected into the cavity, and so was the overlap factor $\beta_{p}=0.14$ [6]. Overlap factor $\beta_{r}$ was obtained from wave calculations considering polar-angle-averaged mode distributions. Finally, the resonance pumping efficiency $\epsilon$ has been measured near $600 \mathrm{~nm}$. By solving Eq. (3) and the relation $\gamma_{r}^{\prime}=\gamma_{r}(1+G)$ simultaneously for two unknowns, we finally obtained the values of $\gamma_{r}$ and $G$ (or equivalently $\left.\bar{g}=\sqrt{\gamma_{r} \gamma_{p} G}\right)$. The results are summarized in Fig. 4(b). The coupling constant $\bar{g}$ is shown to increase as the mode order increases, reflecting the accessibility of the high- $Q$ modes to the chaotic sea. With these values, the resonant pump-coupling efficiencies $\alpha$ and the tunneling rate $\gamma_{r} G$ for all five modes are obtained and summarized in Table I. Note our model discussed so far is also applicable to the scarred modes $(l=4,5)$. In addition, the coupling efficiency about 0.5 has also been confirmed by an independent measurement of the difference between the pumpbeam transmission in the nonresonant pumping and that in the resonant pumping cases [30].

In final remarks, the pump-coupling efficiency $\alpha$ is an inherent quantity for a given cavity whereas the pumping efficiency $\epsilon$ depends on experimental conditions and thus can be further improved. Equation (3) indicates that $\epsilon$ can be increased by up to 1000 times from that of the nonresonant pumping for the $l=1$ mode and about by 600 times for the $l=2$ mode by using a pump laser whose

TABLE I. Pump-coupling efficiency $\alpha$ and the total tunneling rate $\gamma_{r} G$ (in $10^{9} \mathrm{~s}^{-1}$ ) obtained from $\epsilon, \gamma_{r}^{\prime}$ (in $10^{9} \mathrm{~s}^{-1}$ ), and $\beta_{r}$ (with \pm 0.1 error) for all $l=1,2,3,4,5$ modes.

\begin{tabular}{lccccc}
\hline \hline Mode $l$ & $\epsilon$ & $\gamma_{r}^{\prime}$ & $\beta_{r}$ & $\alpha$ & $\gamma_{r} G$ \\
\hline 1 & $25 \pm 4$ & $2.0 \pm 0.6$ & 1.0 & $0.30 \pm 0.16$ & $0.32 \pm 0.18$ \\
2 & $60 \pm 8$ & $7.9 \pm 2.3$ & 0.9 & $0.65 \pm 0.20$ & $3.4 \pm 2.6$ \\
3 & $22 \pm 4$ & $20 \pm 6$ & 0.6 & $0.40 \pm 0.14$ & $4.5 \pm 2.9$ \\
4 & $5.5 \pm 0.7$ & $260 \pm 80$ & 0.4 & $0.44 \pm 0.16$ & $68 \pm 57$ \\
5 & $2.7 \pm 0.4$ & $790 \pm 230$ & 0.3 & $0.62 \pm 0.22$ & $350 \pm 310$ \\
\hline \hline
\end{tabular}

linewidth is much narrower than $\gamma_{r}^{\prime}(\sim \mathrm{GHz})$ of each mode.

In conclusion, we have observed the pump-induced dynamical tunneling in resonant optical pumping of high- $Q$ modes in a significantly deformed microcavity. A mode-mode coupling model was introduced to obtain the resonant pump-coupling efficiencies as well as an effective coupling constant for the tunneling from the observed pumping efficiencies. The present tunneling rate measurement scheme can be readily extended to the quantitative study of dynamical tunneling processes as a function of cavity deformation or size parameter [31], even in the weak coupling regime due to its high sensitivity.

This work was supported by NRL and WCU grants. S. W. K. was supported by a KRF Grant (2008-314C00144). S. Y. L. was supported by the BK21 program.

*kwan@phya.snu.ac.kr

[1] Optical Microcavities, edited by K. Vahala (World Scientific, Singapore, 2005).

[2] V. Sandoghdar et al., Phys. Rev. A 54, R1777 (1996).

[3] J. C. Knight et al., Opt. Lett. 22, 1129 (1997).

[4] Y.S. Park and H. Wang, Nature Phys. 5, 489 (2009).

[5] S.-B. Lee et al., Appl. Phys. Lett. 90, 041106 (2007).

[6] J. Yang et al., Appl. Phys. Lett. 93, 061101 (2008).

[7] C. Gmachl et al., Science 280, 1556 (1998).

[8] M. J. Davis and E. J. Heller, J. Chem. Phys. 75, 246 (1981),

[9] S. Tomsovic and D. Ullmo, Phys. Rev. E 50, 145 (1994).

[10] E. Doron and S. D. Frischat, Phys. Rev. Lett. 75, 3661 (1995).

[11] O. Brodier et al., Phys. Rev. Lett. 87, 064101 (2001).

[12] V. A. Podolskiy and E. E. Narimanov, Phys. Rev. Lett. 91, 263601 (2003).

[13] I. Serban and F. K. Wilhelm, Phys. Rev. Lett. 99, 137001 (2007).

[14] A. Bäcker et al., Phys. Rev. Lett. 100, 104101 (2008).

[15] C. Dembowski et al., Phys. Rev. Lett. 84, 867 (2000).

[16] A. Bäcker et al., Phys. Rev. Lett. 100, 174103 (2008).

[17] T. Neicu et al., Phys. Rev. E 63, 026206 (2001).

[18] W. K. Hensinger et al., Nature (London) 412, 52 (2001).

[19] D. A. Steck et al., Science 293, 274 (2001).

[20] G. Hackenbroich and J. U. Nöckel, Europhys. Lett. 39, 371 (1997).

[21] H. Tureci et al., Opt. Express 10, 752 (2002).

[22] V. A. Podolskiy and E. E. Narimanov, Opt. Lett. 30, 474 (2005).

[23] A. Bäcker et al., Phys. Rev. A 79, 063804 (2009).

[24] J. Yang et al., Rev. Sci. Instrum. 77, 083103 (2006).

[25] S. Moon et al., Opt. Express 16, 11007 (2008).

[26] S.-B. Lee et al., Phys. Rev. A 75, 011802(R) (2007).

[27] S.-B. Lee et al., Phys. Rev. Lett. 88, 033903 (2002).

[28] K. An and J. Yang, arXiv:0912.0164.

[29] J. J. Childs et al., in Cavity Quantum Electrodynamics, edited by P. R. Berman (Academic Press, Boston, 1994).

[30] J. Yang et al. (to be published).

[31] S. Löck et al., Phys. Rev. Lett. 104, 114101 (2010). 